\title{
Reorientation of a hexagonal pattern under broken symmetry: The hexagon flip
}

\author{
Christopher Groh, ${ }^{1}$ Reinhard Richter, ${ }^{1}$ Ingo Rehberg, ${ }^{1}$ and F. H. Busse ${ }^{2}$ \\ ${ }^{1}$ Experimentalphysik V, Universität Bayreuth, D-95440 Bayreuth, Germany \\ ${ }^{2}$ Theoretische Physik IV, Universität Bayreuth, D-95440 Bayreuth, Germany
}

(Received 22 December 2006; revised manuscript received 10 September 2007; published 1 November 2007)

\begin{abstract}
An unexpected pattern transition has been found experimentally in the transformation from hexagons to stripes caused by an applied anisotropy effect. The particular system studied is the surface instability of a horizontal layer of magnetic liquid in a tilted magnetic field. Two orthogonal Helmholtz pairs of coils provide a vertical and a tangential magnetic field. Whereas the vertical component destabilizes the flat layer, the tangential one preserves its stability. The ensuing surface patterns comprise regular hexagons, anisotropic hexagons, and stripelike ridges. The phase diagram for the tilted field instability is measured using a radioscopic technique. The investigation reveals an interesting effect: the flip from one hexagonal pattern to another under an increasing tangential field component, which is explained in terms of amplitude equations as a saddle-node bifurcation.
\end{abstract}

DOI: $10.1103 /$ PhysRevE.76.055301

PACS number(s): 47.65.Cb, 47.54.-r, 47.55.P-

Hexagonal patterns are a common feature in the evolution of instabilities of continuous systems that are isotropic in two dimensions [1]. In systems that are still homogeneous in two dimensions, but without isotropy, instabilities typically exhibit stripelike patterns. Under increasing anisotropy a transition from hexagonal to stripelike patterns can be observed. We report here an intriguing type of pattern transition within this scenario.

Symmetry breaking of hexagonal patterns through anisotropy has been reported in early convection experiments [2], in a chemical oscillator [3], in sorted patterned ground caused by freeze-thaw cycles [4], and in laser experiments [5-8]. In some cases states like "stretched" hexagons were observed, but the underlying bifurcation scenarios remained unclear. This is mainly because of experimental difficulties in tuning the anisotropy at will.

This has become possible in a recent experiment [9] where a magnetic field acts on a layer of ferrofluid, a colloidal dispersion of magnetic nanoparticles [10]. In the ideal horizontally isotropic system, hexagons, as presented in Fig. 1(a) will occur under the influence of a magnetic field that is perfectly normal with respect to the fluid surface [11]. The slightest change of the orientation of the field is predicted [12] to change the regular hexagonal lattice to "stretched" hexagons, as can be seen in Fig. 1(b). For even larger field inclination only liquid ridges along the favored directions remain, as shown in Fig. 1(c). For this transition it was assumed [12] that one of the vectors of the hexagonal lattice is already colinear with the favored direction along the horizontal field component $B_{x}$. Due to the isotropy of the hexagonal pattern, this is not generic, however. In the following we present a transition-namely, the reorientation of the hexagonal pattern in the course of increasing anisotropy. For that purpose the surface reliefs are quantitatively characterized via help of a radioscopic technique.

Our experimental setup is shown in Fig. 2. In the center is a rectangular vessel machined from Perspex. The inner dimensions are $100 \mathrm{~mm}(x), 120 \mathrm{~mm}(y), 25 \mathrm{~mm}(z)$. It is filled up to a brim, fabricated at $z=3 \mathrm{~mm}$, with magnetic fluid mark APG 512 A (Ferrotec Co.). The vessel is fixed in the center of two Helmholtz pairs of coils, which are oriented orthogonally to each other. Each coil generates a component of the applied field tilted to the magnetic fluid surface. An $\mathrm{x}$-ray tube is mounted above the center of the vessel at a distance of $1606 \mathrm{~mm}$. We measure the attenuation of $\mathrm{x}$ rays passing the magnetic fluid layer in the vertical direction with a photodiode array detector (16 bits). The full surface topography is then reconstructed from the calibrated radioscopic images. For details see Ref. [13].

Due to the separate pairs of field coils, we are able to control the induction components independently. First we generate an isotropic hexagonal pattern by increasing $B_{z}$, with $B_{x}$ fixed to $0 \mathrm{mT}$. For this normal field instability [11] the critical induction is surpassed at $B_{c}=16.76 \mathrm{mT}$, resulting in a hexagonal pattern of crests on the magnetic fluid. Figure 3(a) gives the surface topography, where the tips of the crests (red) are positioned in the center of the elevations. In Fourier space all modes have the same amplitude. As indicated in Fig. 4 we denote by $T$ the mode oriented transversally to the horizontal induction component $B_{x}$ and by $C$ the other modes contributing to the hexagon with $T$.

Next we are breaking the symmetry by increasing $B_{x}$, which modifies the hexagons, as shown in Fig. 3(b). The tip of the crests is then tilted towards the direction of $B_{x}$. Increasing $B_{x}$ further gives rise to a reorientation of the hexagonal pattern. Starting at the edges, as shown in Fig. 3(c), all hexagons have flipped in Fig. 3(d) by $30^{\circ}$. In Fourier
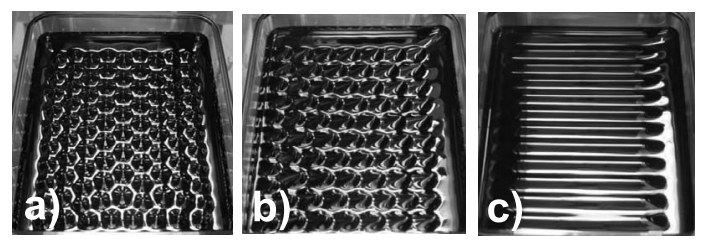

FIG. 1. Surface protuberances on magnetic fluid, where the view is directed along the $y$ axis. Hexagonal pattern for the vertical induction $B_{z}=19.4 \mathrm{mT}$ (a). Increasing the horizontal component from zero to $B_{x}=7.7 \mathrm{mT}$ results in stretched hexagons (b) and finally at $B_{z}=22.4 \mathrm{mT}$ in ridges $(\mathrm{c})$. 


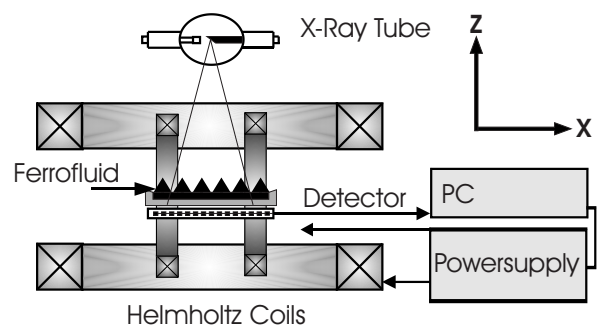

FIG. 2. Scheme of the experimental setup with two Helmholtz pairs of coils for the vertical and horizontal components of the induction, $B_{z}$ and $B_{x}$, respectively.

space the transition is more clearly visible. The prominent longitudinal mode $L$ (cf. Fig. 4) appears first in Fig. 3(c) and is followed by the less dominating mode $D$ in Fig. 3(d). This transition will be referred to as a hexagon flip. A further increase of $B_{x}$ is weakening the $D$ mode until only the $L$ mode survives, as presented in Fig. 3(f). It describes the final pattern of liquid ridges.

When the amplitudes of the selected modes are plotted versus $B_{x}$ the diagram in Fig. 5 results. At low field values the modes $C$ and $T$ are dominating. Both amplitudes decrease by increasing the horizontal field, which in turn is increasing the $L$ mode. At a threshold the $C$ and $T$ modes disappear and the $D$ mode has a flare, until finally only the $L$ mode is left.

A precondition for the hexagon flip is a less than optimal alignment of the primary hexagonal pattern with the direction of anisotropy. Starting from an $L$ hexagon, which is already properly aligned, the flip is not observed. We can prepare such a start configuration in the experiment by starting from liquid ridges and adiabatically reducing $B_{x}$ to zero. Increasing $B_{x}$ again results in the transition of Fig. 6, which shows a simple, monotonous decay of the $D$ mode, in accordance with the model of Ref. [12].

In order to check whether the hexagon flip is a robust phenomenon, we measured it for different values of $B_{z}$ and estimated the induction $B_{x}$ for which $C=D$. The results are

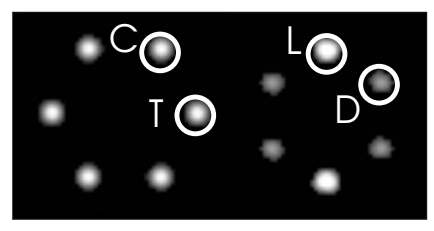

FIG. 4. The transversal $(T)$ and longitudinal $(L)$ modes and the other contributing modes before $(C)$ and after $(D)$ the hexagon flip.

marked by open circles in Fig. 7. To guide the eyes the points are connected by lines (dashed line). The other boundaries were measured by fixing $B_{x}$ and changing $B_{z}$, in this way avoiding a hexagon flip. The solid (dotted) lines denote the bifurcation points for an increase (decrease) of $B_{x}$, respectively. The critical values for the forward bifurcation to ridges have been extracted by fitting the square-root-like evolution of the amplitudes.

For the secondary bifurcation to stretched hexagons the Fourier-domain-based angular correlation function [cf. Eqs. (3)-(6) in [14]) was examined to surpass a threshold of 0.25 of the hexagon amplitude at $60^{\circ}$. The obtained phase diagram displays for $B_{x} \rightarrow 0 \mathrm{mT}$ a narrowing zone of liquid ridges (see Fig. 7) in agreement with theory [12] and a coarser experiment [9]. Similarly the zone of the $L$ hexagons (enclosed by the two upper curves) is narrowing. Note the bistability of $T$ and $L$ hexagons left of the dashed line.

In order to shed some light on the nature of the hexagon flip we present a weakly nonlinear analysis of the competition between hexagonal patterns in an infinitely extended horizontal layer. In the case of horizontal isotropy there is no preferred orientation for a hexagonal pattern, but as soon as a slight homogeneous anisotropy is introduced as by the $x$ component of the applied magnetic field, two steady hexagon patterns are distinguished by their symmetry. The pattern characterized by the (transverse) roll component with wave vector in the $x$ direction consists of $T$ hexagons (see Fig. 4) while the other pattern contains $L$ hexagons. The two other roll components in the hexagon patterns are described by

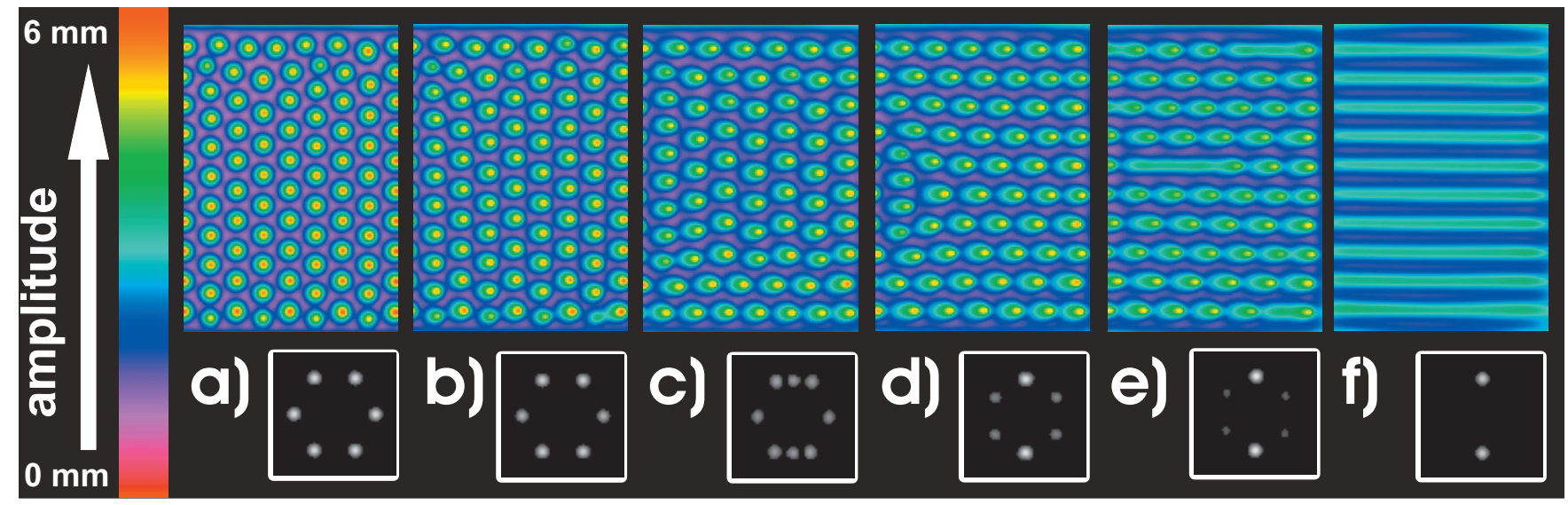

FIG. 3. (Color) Series of surface profiles recorded by the radioscopic technique. The surface elevation is color coded by hue, saturation, value (HSV). $H$ is increased proportional to the height from zero to 360 degree, whereas $S$ and $V$ were fixed to $100 \%$. The horizontal component $B_{x}$ of the induction is oriented towards the right. Underneath, the surface patterns are shown in Fourier space. The vertical induction is fixed to $B_{z}=18.6 \mathrm{mT}$. The horizontal induction $B_{x}$ increases from (a) $3.0 \mathrm{mT}$, via (b) $3.8 \mathrm{mT}$, (c) $5.3 \mathrm{mT}$, (d) $5.8 \mathrm{mT}$, (e) $6.4 \mathrm{mT}$, to (f) $7.7 \mathrm{mT}$. 


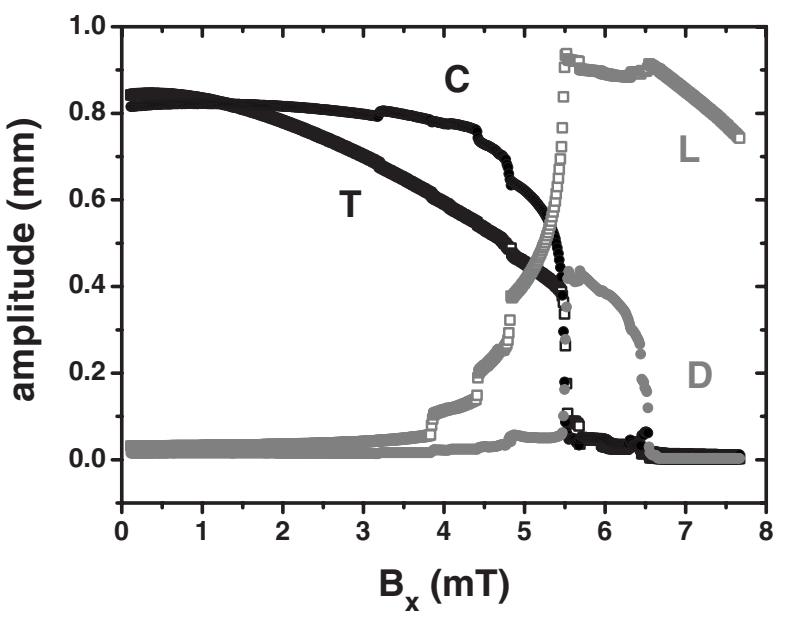

FIG. 5. The measured amplitudes $C$ (black solid circles), $T$ (black open squares), $L$ (gray open squares), and $D$ (gray solid circles) for increasing $B_{x}$.

wave vectors in the directions $(1 / 2, \pm \sqrt{3 / 4})$ in the case of the $T$ hexagons and in the directions $(\sqrt{3 / 4}, \pm 1 / 2)$ in the case of $L$ hexagons. For reasons of symmetry the amplitudes of the two oblique roll components must be equal. In describing the weakly nonlinear states of the two hexagonal patterns and their interactions we are led to the following system of coupled amplitude equations:

$$
\begin{aligned}
\dot{C}= & (\varepsilon-h) C+\eta T C-\left[\gamma\left(2 D^{2}+L^{2}+2 C^{2}+T^{2}\right)\right. \\
& \left.+\delta\left(C^{2}+T^{2}\right)+\lambda\left(D^{2}+L^{2}\right)+\mu D^{2}\right] C, \\
\dot{T}= & (\varepsilon-4 h) T+\eta C^{2}-\left[\gamma\left(2 D^{2}+L^{2}+2 C^{2}+T^{2}\right)\right. \\
& \left.+\delta 2 C^{2}+\lambda 2 D^{2}+\mu L^{2}\right] T,
\end{aligned}
$$

$$
\begin{aligned}
\dot{D}= & (\varepsilon-3 h) D+\eta L D-\left[\gamma\left(2 D^{2}+L^{2}+2 C^{2}+T^{2}\right)\right. \\
& \left.+\delta\left(D^{2}+L^{2}\right)+\lambda\left(C^{2}+T^{2}\right)+\mu C^{2}\right] D,
\end{aligned}
$$

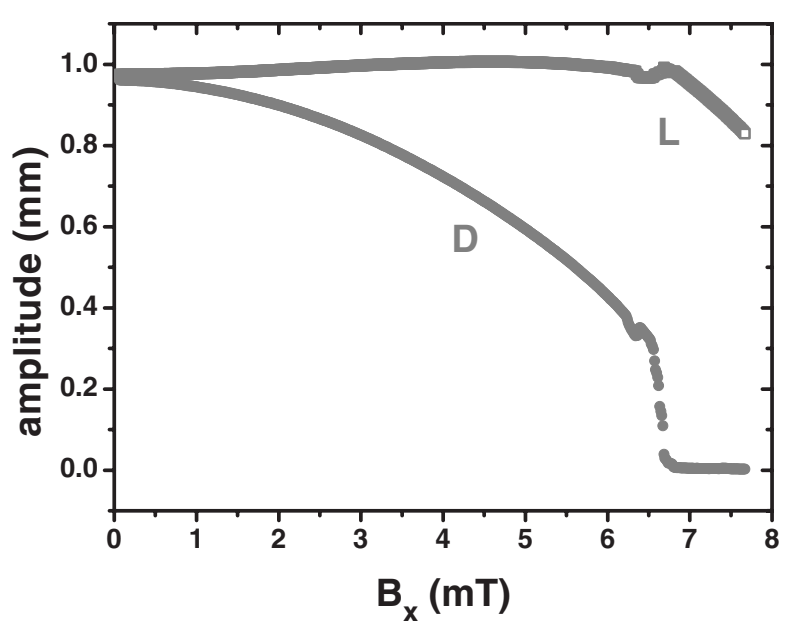

FIG. 6. Evolution of the amplitudes $L$ and $D$ under an increase of $B_{x}$ for hexagons already properly aligned at $B_{x}=0 \mathrm{mT}$.

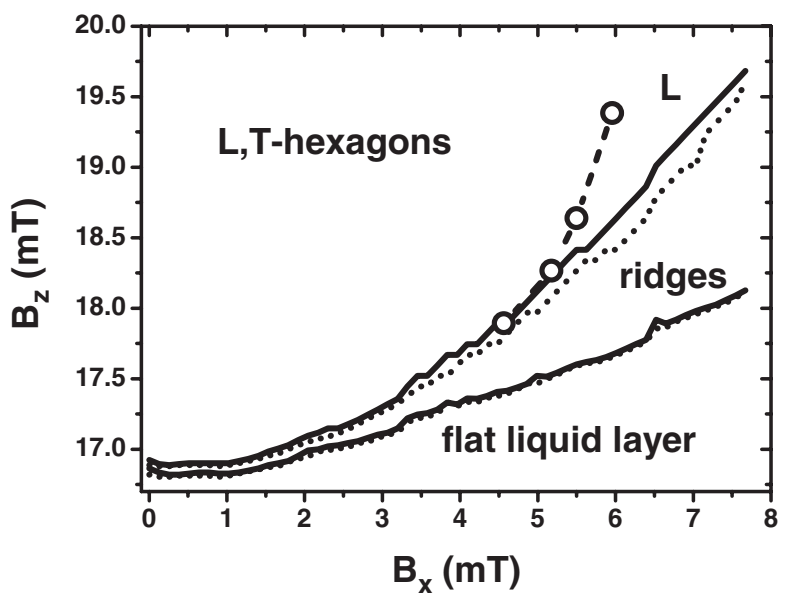

FIG. 7. Phase diagram for the transition to liquid ridges and to hexagons upon increase (solid line) and decrease (dotted line) of $B_{z}$ in steps of $75 \mu T$ for 60 different values of $B_{x}$. The open circles are indicating the hexagon flip for increasing $B_{x}$ and fixed $B_{z}$. To guide the eyes the latter are connected by lines (dashed line).

$$
\begin{aligned}
\dot{L}= & \varepsilon L+\eta D^{2}-\left[\gamma\left(2 D^{2}+L^{2}+2 C^{2}+T^{2}\right)\right. \\
& \left.+\delta 2 D^{2}+\lambda 2 C^{2}+\mu T^{2}\right] L,
\end{aligned}
$$

where $\varepsilon$ is proportional to the supercritical portion of the control parameter in the isotropic case. The stabilizing influence of the homogeneous anisotropy in the $x$ direction will be proportional to the square of the $x$ component of the wave vector in first approximation. The longitudinal roll part described by the amplitude $L$ thus remains unaffected, while the transverse roll component described by the amplitude $T$ suffers the maximum stabilizing influence given by $4 h$ in Eq. (1b). The oblique roll components with amplitudes $D(C)$ of the $L(T)$ hexagons are stabilized by $3 / 4(1 / 4)$ of that amount, respectively.

In the isotropic limit $h=0$, the two steady hexagon solutions $D=L, C=T=0$ and $C=T, D=L=0$ are stable if $\eta \sqrt{\varepsilon}$ is sufficiently large. As $h$ increases the longitudinal roll solution $L^{2}=\varepsilon / \gamma, D=C=T=0$ will eventually become the only stable steady solution, but dependent on the values of the coefficients $\eta, \gamma, \delta, \lambda$, and $\mu$, other solutions may become stable for an intermediate range of $h$. While the system of equations. (1) is universal in that it applies to all systems exhibiting spontaneous hexagonal patterns in the weakly nonlinear limit, the coefficients will depend on the particular realization. Unfortunately coefficients for the Rosensweig instability are not available. We turn to Rayleigh-Bénard convection in an electrically conducting fluid with nonBoussinesq effects as a proxy problem. Here as well as in other situations exhibiting the spontaneous formation of hexagonal patterns we may expect the phenomenon of the hexagon flip. In the case of a convection layer with stress-free and isothermal boundaries, values for $\gamma, \delta, \lambda$, and $\mu$ have been derived $[15,16]$. Using the limit of infinite Prandtl number and setting $\varepsilon=1$ without losing generality one gets $\gamma=1.0$, $\delta=0.5676, \lambda=0.2141$, and $\mu=0.4228$. The parameter $h$ describes the effect of a horizontal magnetic field on convec- 
tion in an electrically conducting fluid, whereas $\eta$ depends on the magnitude of the non-Boussinesq effects and was chosen to be $\eta=5.5$.

Integration in time of Eqs. (1) yields either the $L$-hexagon solution or the upper branch of the $T$-hexagon solution as asymptotic steady states depending on initial conditions. Through the superposition of noise it can be tested that both solutions are stable within Eqs. (1).

It turns out, however, that the $T$-hexagon solution always is replaced at its saddle node by the longitudinal roll solution without an intermediary $L$-hexagon solution. Only when we introduce a slight preference for the $L$-hexagon solution by introducing $\varepsilon=1.2$ in Eqs. (1c) and (1d) instead of $\varepsilon=1$ does the $L$-hexagon pattern appear with a finite amplitude $D$ as demonstrated in Fig. 8. This assumption of different threshold values for $L$ and $T$ hexagons can be justified by the observation that in the experiment the amplitude of the $L$ hexagons for $B_{x}=0 \mathrm{mT}$ is slightly larger than those of the $T$ hexagons (cf. Figs. 6 and 5, respectively). Because the container is not exactly quadratic, the absolute value of the wave vector of the $L$ hexagons may be closer to the critical value than that of the $T$ hexagons. A small difference in $k$ is corroborated by Fig. 3. Also indicated in Fig. 8 is the unstable lower branch of the $T$-hexagon solution, obtained through a Newton-Raphson procedure.

To conclude, we have discovered a bifurcation in the transition scenario from hexagons towards stripes, caused by an increasing strength of a homogeneous anisotropy. Two different steady hexagon states-which are experimentally favored by the rectangular layer-are found to be stable for small anisotropy strengths. One of the two states is terminated by a saddle node at a critical strength of anisotropy.

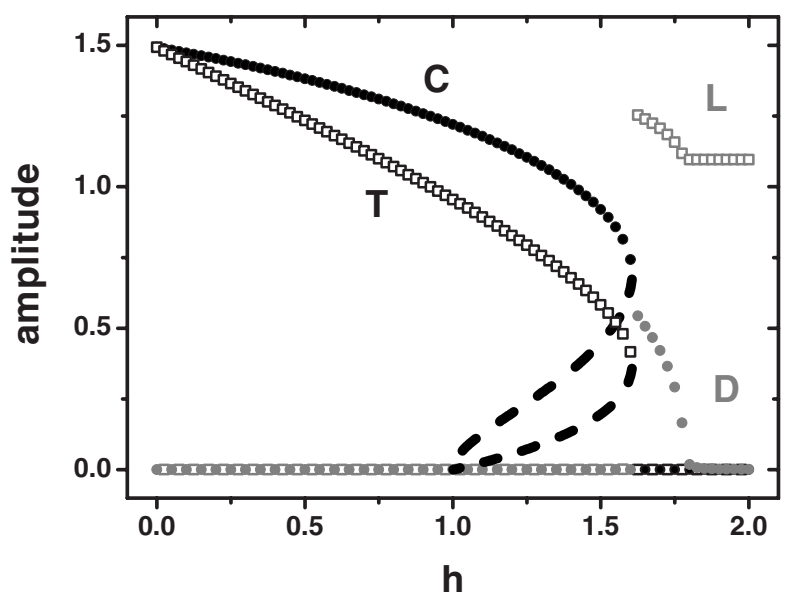

FIG. 8. The calculated amplitudes of the selected modes vs the increasing horizontal induction (cf. Fig. 5). The dashed lines indicate the unstable branches.

The other state continues to evolve into the state of pure stripes with a further increase of the anisotropy strength. The flip transition is of general interest since it may be important for many systems [2-8]. Examples for stretched hexagons of different orientation are reported from laser optics [8]. Moreover, we predict this transition for non-Boussinesq inclinedlayer convection [17] and for convection in electrically conducting fluids or plasmas in magnetic fields.

We thank C. Gollwitzer for valuable advice and discussion. Support by Deutsche Forschungsgemeinschaft via Grant No. Ri 1054/1-4 is gratefully acknowledged.
[1] M. C. Cross and P. C. Hohenberg, Rev. Mod. Phys. 65, 851 (1993).

[2] H. Benard and D. Avsec, J. Phys. Radium 9, 486 (1938).

[3] Q. Ouyang and H. Swinney, Nature (London) 352, 610 (1991).

[4] M. A. Kessler and B. T. Werner, Science 299, 380 (2003).

[5] P. L. Ramazza, S. Bocaletti, and F. T. Arecchi, Opt. Commun. 136, 0267 (1997).

[6] M. Möller et al., Chaos, Solitons Fractals 10, 675 (1999).

[7] T. Ackemann, B. Giese, B. Schäpers, and W. Lange, J. Opt. B: Quantum Semiclassical Opt. 1, 70 (1999).

[8] A. V. Mamaev and M. Saffman, Opt. Lett. 70, 357 (1999).

[9] B. Reimann, R. Richter, H. Knieling, R. Friedrichs, and I. Rehberg, Phys. Rev. E 71, 055202(R) (2005).
[10] R. E. Rosensweig, Ferrohydrodynamics (Cambridge University Press, Cambridge, England, 1985).

[11] M. D. Cowley and R. E. Rosensweig, J. Fluid Mech. 30, 671 (1967).

[12] R. Friedrichs, Phys. Rev. E 66, 066215 (2002).

[13] R. Richter and J. Bläsing, Rev. Sci. Instrum. 72, 1729 (2001).

[14] C. Gollwitzer, I. Rehberg, and R. Richter, J. Phys.: Condens. Matter 18, 2643 (2006).

[15] A. Schlüter, D. Lortz, and F. Busse, J. Fluid Mech. 23, 129 (1965).

[16] F. H. Busse, J. Fluid Mech. 30, 625 (1962).

[17] D. W. Ruth, K. G. T. Hollands, and D. D. Raithby, J. Fluid Mech. 96, 461 (1980). 